\title{
Treatment of nonlinear optical properties due to large amplitude anharmonic vibrational motions: Umbrella motion in $\mathrm{NH}_{3}$
}

\author{
Josep M. Luis, ${ }^{1, a)}$ Heribert Reis, ${ }^{2}$ Manthos Papadopoulos, ${ }^{2}$ and Bernard Kirtman ${ }^{3}$ \\ ${ }^{1}$ Department of Chemistry and Institute of Computational Chemistry, University of Girona, \\ Campus de Montilivi, 17071 Girona, Catalonia, Spain \\ ${ }^{2}$ Institute of Organic and Pharmaceutical Chemistry, National Hellenic Research Foundation, \\ 48 Vas. Constantinou Avenue, 11635 Athens, Greece \\ ${ }^{3}$ Department of Chemistry and Biochemistry, University of California, Santa Barbara, \\ California 93106, USA
}

(Received 8 May 2009; accepted 17 June 2009; published online 21 July 2009)

\begin{abstract}
A general reduced dimensionality finite field nuclear relaxation method for calculating vibrational nonlinear optical properties of molecules with large contributions due to anharmonic motions is introduced. In an initial application to the umbrella (inversion) motion of $\mathrm{NH}_{3}$ it is found that difficulties associated with a conventional single well treatment are overcome and that the particular definition of the inversion coordinate is not important. Future applications are described.

(c) 2009 American Institute of Physics. [DOI: 10.1063/1.3171615]
\end{abstract}

\section{INTRODUCTION}

Nonlinear optical ${ }^{1}$ processes play an important role in scientific and technological fields such as materials science, communications, and medicine. ${ }^{2}$ At the molecular level both the electronic and nuclear degrees of freedom can interact with an electromagnetic light wave and, as a result, nonlinear optical (NLO) properties contain both electronic and vibrational contributions. ${ }^{3-6}$ Surprisingly, for many NLO processes the vibrational contribution is often comparable in magnitude, or even larger, than that due to electronic motions.

A successful method for evaluating the vibrational contribution to NLO properties was formulated about two decades ago by Bishop and Kirtman using a perturbation theory (BK-PT) approach. ${ }^{7-9}$ Much more recently a variational method based on analytical response theory, applied initially to linear polarizabilities, was proposed by Christiansen et al. ${ }^{10,11}$ In either case, for large molecules or very accurate $a b$ initio treatments, both methods are limited in applicability by the major computational effort needed to calculate the potential energy and electrical property surfaces for many nuclear degrees of freedom. This problem can be ameliorated by utilizing the finite field-nuclear relaxation (FF-NR) approach $^{12-16}$ which relies on determining the relaxation of the equilibrium molecular geometry induced by a static electric field. ${ }^{17}$ Indeed, if the leading terms of BK-PT are sufficient, then the FF-NR procedure requires only the electronic (hyper)polarizabilities at the field-free and relaxed geometries. ${ }^{14}$

In principle, the remainder of the vibrational contribution is determined by the zero-point vibrational average (ZPVA) of the (hyper)polarizabilities at field-dependent optimized geometries. ${ }^{15}$ To that end it is necessary to obtain

\footnotetext{
${ }^{a)}$ Author to whom correspondence should be addressed. Electronic mail: josepm.luis@udg.edu.
}

vibrational wave functions associated with anharmonic potential energy surfaces (PESs). For molecules containing large amplitude anharmonic motions, the vibrational wave functions must be obtained by variational (or related) methods since perturbation theory treatments, such as BK-PT, may converge poorly or not at all. ${ }^{18-20}$ Even within a variational treatment, a simple power series expansion of the PES in normal coordinates will not suffice when the anharmonicity is large. ${ }^{21}$ Thus, an accurate numerical description of the potential energy surface is also required, which makes the calculations feasible only for small molecules.

Typically, we expect that only a limited number of internal coordinates will play a significant role in determining vibrational NLO properties, especially when these properties are large. ${ }^{19,21-24}$ One particular example is the case of a molecule with one or a few motions governed by a potential with multiple minima separated by low barriers. Then, one may consider calculating the NLO properties by separating off these motions from all others, and treating the remainder in a simpler fashion. The dimensionality of the vibrational problem for the large amplitude modes will be the same as one would encounter in treating a relatively small molecule. Recently, Santiago et al. ${ }^{4}$ presented a first treatment of vibrational NLO properties along these general lines based on BK-PT (Refs. 7-9) and directed specifically at the torsional motion in hydrogen peroxide. Several approximations were utilized including some for that particular case. In this paper we take a somewhat different approach aimed ultimately at treating both large and small molecules. Our method takes advantage of recent developments that combine FF-NR methodology with variational calculation of field-dependent ZPVA energies and (hyper)polarizabilities. ${ }^{21,25}$ The eventual goal is a practical treatment of vibrational NLO properties that can be applied routinely, and with reliable accuracy, to molecules that may contain several vibrational modes associated with large anharmonic effects. For large molecules the presence of such modes could, in a perverse way, be advan- 
tageous since they will tend to be strongly dominant. Of course, one challenge that will ultimately have to be met is to define a procedure for identifying the set of (potentially) key modes beforehand.

The situation considered here is that of a molecule with a single large amplitude anharmonic mode. For illustrative purposes we present our FF-NR method in terms of the double well potential of ammonia which has two equivalent minima. The quasiegeneracy of the two lowest vibrational states is taken into account using an approach based on generalized Van Vleck perturbation theory (GVV-PT). ${ }^{26-29} \mathrm{Cou}-$ pling to the remaining "spectator" coordinates is eliminated in lowest order by means of nuclear relaxation and ignored in higher order. Thus, our approach completely reduces the calculation of vibrational nonlinear optical properties to an effective one-dimensional (1D) problem. Although $\mathrm{NH}_{3}$ may be considered a simple case it does allow us to perform initial tests of the general strategy and to compare with recent work based on an expansion of the potential about one of the minima. $^{21}$

In Sec. II we set out the FF-NR methodology for treating a large amplitude anharmonic vibration, in particular, for a double potential well with two equivalent minima. Then, in Sec. III we provide illustrative calculations for the inversion mode of $\mathrm{NH}_{3}$, including alternative definitions of this mode, and compare our results to those obtained previously for the single well treatment. Finally, in Sec. IV we summarize our conclusions and discuss plans for future developments.

\section{THEORY}

\section{A. General FF-NR approach}

In the FF-NR approach the vibrational (hyper)polarizability is the sum of a nuclear relaxation term $\left(P^{\mathrm{nr}}\right)$ and a curvature contribution $\left(P^{c}\right) . P^{\mathrm{nr}}$ and $P^{c}$ in turn, arise from the change in the electronic $\left(P^{e}\right)$ and the ZPVA (hyper)polarizability $\left(P^{z p v a}\right)$ caused by field-induced nuclear relaxation. Finally, $P^{c}$ is the sum of $P^{\mathrm{ZPVA}}$ and all remaining vibrational terms beyond $P^{\mathrm{nr}}$ which are denoted as $P^{c \text {-ZPVA }} \cdot{ }^{12-16}$ The FF-NR approach yields the static $P^{\text {nr }}, P^{\text {ZPVA }}$, and $P^{c \text {-ZPVA }}$, as well as $P^{\text {nr }}$ and $P^{c \text {-ZPVA }}$ for the dynamic Pockels effect, $\beta(-\omega ; \omega, 0)$, Kerr effect, $\gamma(-\omega ; \omega, 0,0)$, electric fieldinduced harmonic generation, $\gamma(-2 \omega ; \omega, \omega, 0)$, and degenerate four-wave mixing $\gamma(-\omega ; \omega,-\omega, \omega){ }^{16}$ The latter is also known as the intensity-dependent refractive index. Static vibrational (hyper)polarizabilities are obtained by simple numerical differentiation of the electronic and ZPVA energy (or dipole moment) with respect to the static electric field taking into account the nuclear relaxation. Similarly, the dynamic vibrational NLO properties are obtained by numerical differentiation of the electronic and ZPVA polarizability and first hyperpolarizability. For the dynamic hyperpolarizabilities we use the infinite optical frequency approximation, which is highly accurate at normal laser optical frequencies. ${ }^{23,30,31}$

\section{B. Inversion coordinate}

As pointed out in Sec. I, the application of our finite field approach for $\mathrm{NH}_{3}$ requires calculation of the effective 1D double minimum well potential for inversion. The inversion coordinate must be selected in such a way that it is able to describe the entire range of motion without discontinuities. We chose to investigate two such coordinates for comparison purposes. The first of these, $\theta$, was previously used by Handy et $a .^{32}$ It is given in terms of the three angles $\theta_{1}, \theta_{2}$, and $\theta_{3}$ defined by the $\mathrm{N}-\mathrm{H}$ bonds and the trisector axis. The latter is defined in such a way that the three angles are equal. Then the expression of $\theta$ is given by

$$
\theta=\frac{1}{3}\left(\theta_{1}+\theta_{2}+\theta_{3}\right)-\frac{\pi}{2}
$$

The second coordinate, $z$, is the displacement between the plane defined by the three hydrogens and the $\mathrm{N}$ atom. ${ }^{33}$ This displacement may be either positive or negative.

\section{Effective 1D potential energy function and kinetic energy operator}

The 1D potential energy function, $V\left(q_{\text {inv }}\right)$, was obtained as the electronic energy at a fixed value of the inversion coordinate, $q_{\text {inv }}$, with the geometry otherwise optimized. This eliminates all linear terms in the remaining vibrational degrees which means that the first-order coupling between the inversion coordinate and the remaining spectator coordinates is included in the effective 1D potential.

The next step is to express the kinetic energy operator, $\hat{T}$, in terms of $q_{\text {inv }}$ and derivatives with respect to $q_{\text {inv. }}$. For molecules with more than five atoms the analytical expression for the kinetic energy operator can be very complex. In order to have a general method that can be easily applied to any molecule we decided to evaluate $\hat{T}$ numerically. Two different programs were investigated for this purpose, KICO (Refs. 34 and 35) and TNUM. ${ }^{36,37}$ Both programs require only the Cartesian coordinates of the atoms for any of the usual internal coordinates. However, TNUM readily allows for the definition of linear combinations of internal coordinates as well. As this is a necessary requisite for inversion coordinate $\theta$, TNUM was adopted for this project.

The formal expression for the kinetic energy operator of an $N$ atom molecule as a function of the $3 N$ generalized coordinates $q^{i}(i=1, \ldots, 3 N)$ can be easily derived as ${ }^{36,37}$

$$
\hat{T}(\mathbf{q})=\sum_{i j}^{3 N} f_{2}^{i j}(\mathbf{q}) \partial_{i j}^{2}+\sum_{i}^{3 N} f_{1}^{i}(\mathbf{q}) \partial_{i}+v(\mathbf{q}),
$$

where

$$
\begin{aligned}
& f_{2}^{i j}(\mathbf{q})=-\frac{\hbar^{2}}{2} G^{i j}(\mathbf{q}), \\
& f_{1}^{i}(\mathbf{q})=-\frac{\hbar^{2}}{2} \sum_{j}^{3 N}\left[G^{i j}(\mathbf{q}) \partial_{j} \ln J(\mathbf{q})+\partial_{j} G^{i j}(\mathbf{q})\right],
\end{aligned}
$$

with

$$
G^{i j}(\mathbf{q})=\left[g^{i j}(\mathbf{q})\right]^{-1}
$$

and 


$$
\begin{aligned}
& g^{i j}(\mathbf{q})=\sum_{\lambda}^{3 N}\left(\partial_{i} x^{\lambda}(\mathbf{q})\right)\left(\partial_{j} x^{\lambda}(\mathbf{q})\right), \\
& J(\mathbf{q})=\operatorname{det}(\mathbf{J})=\operatorname{det}\left[\partial_{i} x^{\lambda}\right] .
\end{aligned}
$$

In Eqs. (6) and (7) the quantities $x^{\lambda}(\lambda=1, \ldots, 3 N)$ are the molecule-fixed mass-weighted Cartesian coordinates. Evaluation of the first two terms in Eq. (2) requires only the calculation of the $G$ matrix, the determinant of the Jacobian, and their derivatives. The third term of Eq. (2) is a pseudopotential $^{37}$ which arises from the volume element used in normalizing the wave function. ${ }^{38}$ In this paper we have used the Wilson normalization, ${ }^{38,39}$ which has the advantage that the volume element is simply expressed as

$$
d \tau=d q^{1}, d q^{2}, \ldots, d q^{3 N} .
$$

For each value of $\mathbf{q}$, TNUM computes $f_{2}^{i j}(\mathbf{q}), f_{1}^{i}(\mathbf{q})$, and $v(\mathbf{q})$. Then $\hat{T}(\mathbf{q})$ is obtained immediately from Eq. (2).

Another important advantage of using TNUM is that the same formulation of the kinetic energy operator is valid for reduced dimensionality models if the $\mathbf{G}(\mathbf{q})$ matrix is replaced by the corresponding reduced dimensionality $\mathbf{G}^{\text {red }}(\mathbf{q})$, which is calculated using a procedure ${ }^{37}$ equivalent to the one originally proposed by Wilson et al. ${ }^{39}$ The coordinates not included in the reduced dimensionality model can be frozen or considered as a function of the active coordinates, ${ }^{36,37,40-43}$ which was done here. The reduced dimensionality kinetic operator calculated in this manner is exact. Finally, our results for $f_{2}\left(q_{\text {inv }}\right), f_{1}\left(q_{\text {inv }}\right)$, and $f_{3}\left(q_{\text {inv }}\right)=V\left(q_{\text {inv }}\right)+v\left(q_{\text {inv }}\right)$, obtained for different values of $q_{\text {inv }}$, are fit to a polynomial of the $n$th degree, ${ }^{33}$

$$
f_{i}\left(q_{\mathrm{inv}}\right)=\sum_{j=0}^{n} a_{j}^{i} q_{\mathrm{inv}}^{j} .
$$

The value of $n$ was increased until the desired convergence of the field-dependent vibrational energies was achieved.
This entire procedure was repeated for each value of the electric field which then yields the field-dependent 1D effective inversion Hamiltonian

$$
\hat{H}\left(q_{\text {inv }}, F_{z}\right)=\hat{T}\left(q_{\text {inv }}\right)+V\left(q_{\text {inv }}, F_{z}\right) .
$$

We note that when the electric field is present this operator is no longer symmetric in $q_{\text {inv }}$ and terms that give rise to nuclear relaxation are included.

\section{Evaluation of vibrational (hyper)polarizabilities for a double minimum well potential using a two-state reference space}

As observed in Sec. II A the practical calculation of vibrational NLO properties using the FF-NR method requires differentiation of ZPVA (hyper)polarizabilies and energies (for static properties) with respect to a finite (static) field. In the absence of tunneling the field-free vibrational ground state will be doubly degenerate corresponding to motion along the inversion coordinate in each of the two potential wells. These states will be split by tunneling into a quasidegenerate symmetric and antisymmetric pair. In order to analyze the perturbing effect of a static electric field we utilize a procedure based on generalized Van Vleck quasidegenerate perturbation theory. ${ }^{26-29}$ The model (or reference) space consists of the ground state symmetric and antisymmetric inversion doublet $\varphi^{+}$and $\varphi^{-}$. By applying GVV-PT one reduces the coupling between the model space and all remaining vibrational states to higher order. We assume, as usual, that the higher-order coupling terms can be neglected and also that the resulting Hamiltonian matrix elements for the model space can be written as a power series in the field. Due to inversion symmetry that matrix will have the general form (through fourth order in the field),

$$
\hat{H}\left(F_{z}\right)=\left(\begin{array}{cc}
E^{+}(0)-\frac{1}{2} \alpha_{z z}^{+} F_{z}^{2}-\frac{1}{24} \gamma_{z z z}^{+} F_{z}^{4} & -\mu_{z}^{ \pm} F_{z}-\frac{1}{6} \beta_{z z z}^{ \pm} F_{z}^{3} \\
-\mu_{z}^{ \pm} F_{z}-\frac{1}{6} \beta_{z z z}^{ \pm} F_{z}^{3} & E^{-}(0)-\frac{1}{2} \alpha_{z z}^{-} F_{z}^{2}-\frac{1}{24} \gamma_{z z z z}^{-} F_{z}^{4}
\end{array}\right),
$$

where $+(-)$ indicates the symmetric (antisymmetric) reference state and $F_{z}$ is an electric field along the threefold symmetry axis $(z)$.

The final step in GVV-PT is to diagonalize $\hat{H}\left(F_{z}\right)$ to obtain the vibrational field-dependent energies corresponding to the ground state inversion doublet. If the $\varphi^{+}$and $\varphi^{-}$states are nondegenerate due to tunneling, then the usual formula for the eigenvalues will contain a term that involves the square root of a power series in the field. Consequently, the eigenvalues will not be expandable in such a series except under one of the following two conditions. That is to say, either $\left(E^{+}(0)-E^{-}(0)\right)^{2}$ is negligible in magnitude compared to $\left(2 \mu_{z}^{ \pm} F\right)^{2}$ or vice versa. For $\mathrm{NH}_{3}$ the splitting of the ground state due to inversion is quite small, which means that the former condition will be satisfied if the electric field is not too weak. In Sec. III we show that the strong electric field condition is clearly fulfilled by the field strengths used in our finite field calculations. Indeed, the minimum field strength was determined by increasing the value used in a power series fit of the dipole moment and (hyper)polarizabilities until these properties no longer vary significantly.

We emphasize that the use of GVV-PT in the preceding paragraphs is solely to derive the finite field equations [Eqs. (14)-(17) below] from which the vibrational dipole moment 
and (hyper)polarizabilities are obtained. In actual calculations we obtain the lowest pair of field-dependent energies directly through exact numerical solution of the fielddependent Schrodinger equation (see Sec. II E). The resulting field-dependent vibrational energies automatically contain the effect of coupling between the model space and all remaining vibrational states. Thus, tunneling is completely taken into account. Nevertheless, as in the case of GVV-PT, the utilization of a power series expansion in the field is valid only for the regime where inversion splitting is negligible compared to the first-order (plus third-order) off-diagonal terms in the field.

If the condition for a power series expansion in $F_{z}$ is met, then the eigenvalues of the Hamiltonian matrix in Eq. (11) are given by

$$
\begin{aligned}
& E_{0}(F)=E(0)-\mu_{z} F_{z}-\frac{1}{2} \alpha_{z z} F_{z}^{2}-\frac{1}{6} \beta_{z z z} F_{z}^{3}-\frac{1}{24} \gamma_{z z z z} F_{z}^{4}, \\
& E_{1}(F)=E(0)+\mu_{z} F_{z}-\frac{1}{2} \alpha_{z z} F_{z}^{2}+\frac{1}{6} \beta_{z z z} F_{z}^{3}-\frac{1}{24} \gamma_{z z z z} F_{z}^{4},
\end{aligned}
$$

where $\quad E(0)=\frac{1}{2}\left[E^{+}(0)+E^{-}(0)\right], \quad \alpha_{z z}=\frac{1}{2}\left[\alpha_{z z}^{+}+\alpha_{z z}^{-}\right], \quad \gamma_{z z z z}$ $=\frac{1}{2}\left[\gamma_{z z z z}^{+}+\gamma_{z z z z}^{-}\right], \mu_{z}=\mu_{z}^{ \pm}$, and $\beta_{z z z}=\beta_{z z z}^{ \pm}$. Clearly, it is sufficient to utilize only positive fields to determine $E(0), \mu_{z}, \alpha_{z z}$, etc. Based on the convention that $\mu_{z}$ is positive $E_{0}(F)$ will then always correspond to the lower energy state. From Eqs. (12) and (13) the diagonal $z$ component of the static dipole moment and (hyper)polarizabilities can be obtained using the numerical differentiation formulas,

$$
\begin{aligned}
& \mu_{z}=\frac{-E_{1}\left(2 F_{z}\right)+E_{0}\left(2 F_{z}\right)+8 E_{1}\left(F_{z}\right)-8 E_{0}\left(F_{z}\right)}{12 F_{z}}, \\
& \alpha_{z z}(0 ; 0)=\frac{E_{1}\left(4 F_{z}\right)+E_{0}\left(4 F_{z}\right)-17 E_{1}\left(2 F_{z}\right)-17 E_{0}\left(2 F_{z}\right)+16 E_{1}\left(F_{z}\right)+16 E_{0}\left(F_{z}\right)}{36 F_{z}^{2}}, \\
& \beta_{z z z}(0 ; 0,0)=\frac{E_{1}\left(2 F_{z}\right)-E_{0}\left(2 F_{z}\right)-2 E_{1}\left(F_{z}\right)+2 E_{0}\left(F_{z}\right)}{2 F_{z}^{3}}, \\
& \gamma_{z z z z}(0 ; 0,0,0)=\frac{-E_{1}\left(4 F_{z}\right)-E_{0}\left(4 F_{z}\right)+5 E_{1}\left(2 F_{z}\right)+5 E_{0}\left(2 F_{z}\right)-4 E_{1}\left(F_{z}\right)-4 E_{0}\left(F_{z}\right)}{15 F_{z}^{4}} .
\end{aligned}
$$

Since the above expressions give the derivatives of the total 1D field-dependent electronic energy averaged over the 1D field-dependent vibrational wave functions, they include all electronic and vibrational contributions within the 1D (reduced dimensionality) approximation. This treatment corresponds essentially to combining the Bishop-Hasan-Kirtman (BHK) (Ref. 12) and Kirtman-Luis-Bishop (KLB) (Ref. 15) FF-NR procedures into a single step. In those papers the static properties were obtained from the dipole moment, but the same procedure has since been employed using the energy instead. ${ }^{25}$ The total property value may be written as the sum of four terms: $P=P^{e}+P^{\mathrm{nr}}+P^{\mathrm{ZPVA}}+P^{c \text {-ZPVA }}$. Since the first three of these are readily obtained separately $\left(P^{\text {nr }}\right.$ by the standard FF-NR method ${ }^{12-14}$ and $P^{\mathrm{ZPVA}}$ as in Sec. II E) we can use the total $P$ to determine $P^{c \text {-ZPVA }}$. In order to crosscheck our results, the static (hyper)polarizabilities were also calculated from the corresponding numerical differentiation formulas for the dipole moment.

By proceeding in the same vein, dynamic hyperpolarizabilities can be determined from calculations of the fielddependent (static) polarizability and first hyperpolarizability averaged over the field-dependent vibrational wave functions. Numerical differentiation, cf. Eqs. (14) and (15), then yields the following combined BHK and KLB expressions:

$$
\begin{aligned}
& \beta_{z z z}^{e}(0 ; 0,0)+\beta_{z z z}^{\mathrm{ZPVA}}(0 ; 0,0)+\beta_{z z z}^{\mathrm{nr}}(-\omega ; \omega, 0)_{\omega \rightarrow \infty}+\beta_{z z z}^{c-\mathrm{ZPVA}}(-\omega ; \omega, 0)_{\omega \rightarrow \infty} \\
& =\frac{-\alpha_{z z, 1}\left(2 F_{z}\right)+\alpha_{z z, 0}\left(2 F_{z}\right)+8 \alpha_{z z, 1}\left(F_{z}\right)-8 \alpha_{z z, 0}\left(F_{z}\right)}{12 F_{z}}, \\
& \gamma_{z z z z}^{e}(0 ; 0,0,0)+\gamma_{z z z z}^{\mathrm{ZPVA}}(0 ; 0,0,0)+\gamma_{z z z z}^{\mathrm{nr}}(-\omega ; \omega, 0,0)_{\omega \rightarrow \infty}+\gamma_{z z z z}^{c-\mathrm{ZPV}}(-\omega ; \omega, 0,0)_{\omega \rightarrow \infty} \\
& \quad=\frac{\alpha_{z z, 1}\left(4 F_{z}\right)+\alpha_{z z, 0}\left(4 F_{z}\right)-17 \alpha_{z z, 1}\left(2 F_{z}\right)-17 \alpha_{z z, 0}\left(2 F_{z}\right)+16 \alpha_{z z, 1}\left(F_{z}\right)+16 \alpha_{z z, 0}\left(F_{z}\right)}{36 F_{z}^{2}},
\end{aligned}
$$




$$
\begin{aligned}
& \gamma_{z z z z}^{e}(0 ; 0,0,0)+\gamma_{z z z z}^{\mathrm{ZPA}}(0 ; 0,0,0)+\gamma_{z z z z}^{\mathrm{nr}}(-2 \omega ; \omega, \omega, 0)_{\omega \rightarrow \infty}+\gamma_{z z z z}^{c-\mathrm{ZPVA}}(-2 \omega ; \omega, \omega, 0)_{\omega \rightarrow \infty} \\
& \quad=\frac{-\beta_{z z z, 1}\left(2 F_{z}\right)+\beta_{z z z, 0}\left(2 F_{z}\right)+8 \beta_{z z z, 1}\left(F_{z}\right)-8 \beta_{z z z, 0}\left(F_{z}\right)}{12 F_{z}},
\end{aligned}
$$

where $\alpha_{z z, 0}\left(F_{z}\right)$ and $\alpha_{z z, 1}\left(F_{z}\right)\left(\beta_{z z z, 0}\left(F_{z}\right)\right.$ and $\left.\beta_{z z, 1}\left(F_{z}\right)\right)$ are the field-dependent static polarizabilities (first hyperpolarizability). We determine $\beta_{z z z}^{\mathrm{nr}}(-\omega ; \omega, 0)_{\omega \rightarrow \infty}, \gamma_{z z z z}^{\mathrm{nr}}(-\omega ; \omega, 0,0)_{\omega \rightarrow \infty}$, and $\gamma_{z z z z}^{\mathrm{nr}}(-2 \omega ; \omega, \omega, 0)_{\omega \rightarrow \infty}$ by means of the standard FF-NR method and, then, the corresponding $c$-ZPVA (hyper)polarizabilities are obtained by subtracting these terms, along with the static electronic and ZPVA contributions, from the total values given by Eqs. (18)-(20).

\section{E. Solution of the 1D field-dependent vibrational Schrödinger equation}

In order to obtain the field-dependent energies required by our finite field method we have to solve the fielddependent 1D vibrational Schrödinger equation for the ground and first exited states at each value of $F_{z}$,

$$
\begin{aligned}
\hat{H}\left(q_{\mathrm{inv}}, F_{z}\right) \psi_{i}\left(q_{\mathrm{inv}}, F_{z}\right)= & \hat{T}\left(q_{\mathrm{inv}}\right) \psi_{i}\left(q_{\mathrm{inv}}, F_{z}\right) \\
& +V\left(q_{\mathrm{inv}}, F_{z}\right) \psi_{i}\left(q_{\mathrm{inv}}, F_{z}\right) \\
= & E_{i}\left(F_{z}\right) \psi_{i}\left(q_{\mathrm{inv}}, F_{z}\right) .
\end{aligned}
$$

Solutions were obtained by means of the so-called shooting method. ${ }^{44}$ The procedure involves writing the second-order ordinary differential equation as a coupled pair of first-order differential equations. In our case the wave function must vanish at large negative and positive values of the inversion coordinate. ${ }^{32,33}$ In the shooting method this two point boundary value problem is converted to an initial value problem. Thus, the values of the vibrational wave function and its first derivative (with respect to the inversion coordinate) at the starting point are chosen to fulfill the boundary conditions at that point (i.e., both quantities are set equal zero). Then, the ordinary differential equations are integrated numerically using the Runge-Kutta method. In the first try the final point will normally not fulfill the boundary condition. So the integration is repeated, using a globally convergent Newton's method to adjust values of the vibrational energy, until the final point satisfies the boundary condition as well. In the shooting method the initial guess for the energy determines which state is obtained. In each case we verified that the desired inversion doublet was obtained. Although not necessary, a final check on the orthogonality of the two states was also carried out.

The ZPVA contributions to the dipole moment and (hyper)polarizabilities were computed numerically as expectation values using the field-dependent vibrational wave functions for the inversion doublet, and the appropriate property expression as a function of $q_{\text {inv }}$. These property expressions were obtained in the same fashion as the PES by fitting to a polynomial as in Eq. (9). As a check we compared the fieldfree dipole moment obtained in this manner with the dipole moment obtained from Eq. (14) to make sure that the two agree.

\section{ILLUSTRATIVE EXAMPLE: $\mathrm{NH}_{3}$}

In order to perform a proper comparison with our previous single well treatment ${ }^{21}$ we compute the vibrational (hyper)polarizabilities of $\mathrm{NH}_{3}$ using the same level of electronic structure calculations as before. Thus, the effective 1D potential energy function was computed using the GAUSSIAN03 suite of programs ${ }^{45}$ at the MP2 level with the POL basis set developed by Sadlej ${ }^{46}$ for determination of polarizabilities. Subsequent tests on a number of molecules have confirmed that this basis does, indeed, give satisfactory results for electric field polarization properties. ${ }^{47-51}$ Our first step was to check that MP2/POL is also adequate to describe the vibrational energy levels for the umbrella motion of ammonia. In Table I we compare experimental values ${ }^{52}$ for the first seven excited states with our theoretical results calculated at the MP2/POL, ${ }^{46}$ CCSD/aug-cc-pvTZ, ${ }^{53,54}$ and CCSD(T)/aug-ccpvTZ levels. For MP2/POL both the $\theta$ (Ref. 32) and the $z$ inversion coordinate ${ }^{33}$ were utilized whereas, for CCSD/augcc-pvTZ and $\operatorname{CCSD}(\mathrm{T}) /$ aug-cc-pvTZ, we used only $\theta$. In Table II we present the inversion splittings for the same set of states.

Both $\mathrm{MP} 2 / \mathrm{POL} / \theta$ and $\mathrm{MP} 2 / \mathrm{POL} / z$ reproduce the general trends of the experimental spectra. While the former is clearly better for the energy levels, the latter is more accurate for the splittings. The mean absolute deviation (MAD) and maximum absolute deviation (MAXAD) obtained for the energy levels with $\mathrm{MP} 2 / \mathrm{POL} / \theta$ turns out to be better than the 1D CCSD(T)/aug-cc-pvTZ values obtained by Pesonen et $a{ }^{55}$ using a frozen $\mathrm{N}-\mathrm{H}$ distance. Their MAD and MAXAD are 55.20 and $100.05 \mathrm{~cm}^{-1}$, respectively, compared to our values of 27.60 and $84.23 \mathrm{~cm}^{-1}$. On the other hand, the opposite conclusion applies to the inversion splittings. Their MAD (MAXAD) is $5.22 \mathrm{~cm}^{-1}\left(16.56 \mathrm{~cm}^{-1}\right)$ while ours is $10.22 \mathrm{~cm}^{-1}\left(19.48 \mathrm{~cm}^{-1}\right)$.

The computed energy levels and inversion splittings improve, compared to experiment, as the level of the electronic structure treatment increases from MP2/POL to CCSD/augcc-pvTZ to $\operatorname{CCSD}(\mathrm{T}) /$ aug-cc-pvTZ. Our 1D effective $\operatorname{CCSD}(\mathrm{T}) /$ aug-cc-pvTZ/ $\theta$ approach leads to a MAD (MAXAD) of $12.39 \mathrm{~cm}^{-1}\left(20.08 \mathrm{~cm}^{-1}\right)$ for the energy levels and $14.04 \mathrm{~cm}^{-1}\left(29.63 \mathrm{~cm}^{-1}\right)$ for the inversion splittings. The MP2/POL/ $\theta$ results differ from experiment by roughly twice as much. Nevertheless, they are satisfactory for our purposes which are to (i) illustrate the new methodology and (ii) perform a proper comparison with our previous single well treatment, rather than to calculate benchmark values for comparison with experiment or previous literature results. 
TABLE I. Experimental and calculated vibrational energy levels for the double minimum potential of ammonia in $\mathrm{cm}^{-1}$.

\begin{tabular}{lrrrrr}
\hline \hline State & Experimental $^{\mathrm{a}}$ & $\mathrm{MP2} / \mathrm{POL} / \theta^{\mathrm{b}}$ & $\mathrm{MP} / \mathrm{POL} / \mathrm{z}^{\mathrm{c}}$ & $\mathrm{CCSD} / \mathrm{aTZ} / \theta^{\mathrm{d}}$ & $\left.\mathrm{CCSD}^{\mathrm{N}}\right) / \mathrm{aTZ} / \theta^{\mathrm{e}}$ \\
\hline $0^{-}$ & 0.79 & 0.37 & 0.47 & 1.27 & 1.07 \\
$1^{+}$ & 932.43 & 970.00 & 1008.94 & 911.38 & 917.26 \\
$1^{-}$ & 968.12 & 989.89 & 1036.09 & 962.68 & 962.31 \\
$2^{+}$ & 1598.47 & 1682.70 & 1721.27 & 1560.46 & 1570.39 \\
$2^{-}$ & 1882.18 & 1905.31 & 1992.45 & 1890.49 & 1883.72 \\
$3^{+}$ & 2384.17 & 2406.68 & 2515.02 & 2405.26 & 2393.64 \\
$3^{-}$ & 2895.61 & 2892.04 & 3045.93 & 2942.74 & 2921.99 \\
MAD $^{\mathrm{f}}$ & & 27.60 & 94.15 & 20.21 & 12.39 \\
MAXAD $^{\mathrm{g}}$ & & 84.23 & 150.32 & 47.13 & 28.08 \\
\hline \hline
\end{tabular}

\footnotetext{
${ }^{a}$ Experimental data taken from Ref. 52.

${ }^{\mathrm{b}}$ Results obtained using $\theta$ inversion coordinate 1D PES calculated at MP2/POL level (Ref. 46).

${ }^{c}$ Results obtained using $z$ inversion coordinate 1D PES calculated at MP2/POL level (Ref. 46).

${ }^{\mathrm{d}}$ Results obtained using $\theta$ inversion coordinate 1D PES calculated at CCSD/aug-cc-pvTZ level (Refs. 53 and 54).

${ }^{e}$ Results obtained using $\theta$ inversion coordinate 1D PES calculated at CCSD(T)/aug-cc-pvTZ level (Refs. 53 and 54).

${ }^{\mathrm{f}}$ Mean absolute deviation

${ }^{\mathrm{g}}$ Maximum absolute deviation.
}

Although the deviations are significantly larger for the $\mathrm{MP} 2 / \mathrm{POL} / z$ energy levels, which are more important than inversion splittings for vibrational (hyper)polarizabilities, we obtain the MP2/POL properties for $z$ as well as $\varphi$ in order to see how sensitive the results are to the choice of coordinate. Of course, the MAD can be reduced by increasing the dimensionality to two dimensional ${ }^{55}$ and six dimensional ${ }^{56-58}$ Indeed, our third goal is to see the effect of reducing the dimensionality.

In Tables III-V we present the electronic and vibrational contributions to the dipole moment, and to the diagonal component of the (hyper)polarizabilities, along the $C_{3}$ symmetry axis of ammonia. The static electric fields used in the finite field calculations are $\pm 0.0004, \pm 0.0008, \pm 0.0016$, $\pm 0.0032, \pm 0.0064$, and \pm 0.0128 a.u. For the smallest field of magnitude 0.0004 a.u. the ratio $\left(E^{+}(0)\right.$ $\left.-E^{-}(0)\right)^{2} /\left(2 \mu_{z}^{ \pm} F\right)^{2}$ is equal to $1.2 \times 10^{-5}$. Thus, the strong electric field condition is fulfilled in all cases. The most accurate derivatives were selected from the Romberg method triangle ${ }^{59}$ leading to the results in Tables III-V.
Our current calculations confirm two results established in our previous work. ${ }^{21}$ One is that the vibrations often play an essential role in determining the NLO properties of ammonia. For example, in the case of the static first hyperpolarizability (see Table IV) the vibrational contribution $\left(\beta^{\text {nr }}\right.$ $\left.+\beta^{\mathrm{ZPVA}}+\beta^{c-\mathrm{ZPVA}}\right)$ is about 7.5 times larger than the electronic contribution $\left(\beta^{e}\right)$. The other is that the inversion mode strongly dominates the vibrational contribution. Indeed, it is very close to the fourth-order value (see further below) obtained for the complete set of $3 N-6$ normal modes when the vibrational contribution is most important, as for the first and second static hyperpolarizabilities.

Our previous calculations utilized various Taylor series expansions of the PES and of the electronic properties $\left(\mu^{e}\right.$, $\alpha^{e}$, etc.) as a function of the normal coordinates $(\boldsymbol{Q})$ defined in terms of displacements from one of the two minima. ${ }^{21,60,11}$ For a given representation of the PES, low-order expansions of the electronic properties led to converged vibrational NLO properties. However, the same was not true the other way around, i.e., for a given representation of the electronic prop-

TABLE II. Experimental and calculated inversion splittings for ammonia in $\mathrm{cm}^{-1}$.

\begin{tabular}{lccccc}
\hline \hline State & Experimental $^{\mathrm{a}}$ & $\mathrm{MP} 2 / \mathrm{POL} / \theta^{\mathrm{b}}$ & $\mathrm{MP} / \mathrm{POL} / \mathrm{z}^{\mathrm{c}}$ & $\mathrm{CCSD} / \mathrm{aTZ} / \theta^{\mathrm{d}}$ & $\mathrm{CCSD}(\mathrm{T}) / \mathrm{aTZ} / \theta^{\mathrm{e}}$ \\
\hline 0 & 0.79 & 0.37 & 0.47 & 1.27 & 1.06 \\
1 & 35.69 & 19.89 & 27.15 & 51.30 & 45.06 \\
2 & 283.71 & 222.61 & 271.17 & 330.03 & 313.34 \\
3 & 511.44 & 485.36 & 530.92 & 537.48 & 528.35 \\
MAD $^{\mathrm{f}}$ & & 25.85 & 10.22 & 22.11 & 14.04 \\
MAXAD $^{\mathrm{g}}$ & & 61.10 & 19.48 & 46.32 & 29.63 \\
\hline \hline
\end{tabular}

${ }^{\mathrm{a}}$ Experimental data taken from Ref. 52.

${ }^{\mathrm{b}}$ Results obtained using $\theta$ inversion coordinate 1D PES calculated at MP2/POL level (Ref. 46).

${ }^{c}$ Results obtained using $z$ inversion coordinate 1D PES calculated at MP2/POL level (Ref. 46).

${ }^{\mathrm{d}}$ Results obtained using $\theta$ inversion coordinate 1D PES calculated at CCSD/aug-cc-pvTZ level

(Refs. 53 and 54).

${ }^{\text {e }}$ Results obtained using $\theta$ inversion coordinate 1D PES calculated at CCSD(T)/aug-cc-pvTZ level

(Refs. 53 and 54).

${ }^{\mathrm{f}}$ Mean absolute deviation.

${ }^{\mathrm{g}}$ Maximum absolute deviation. 
TABLE III. Electronic and vibrational contributions to $\mu$, and to the diagonal component of $\alpha$ in the direction of $\mu$ for $\mathrm{NH}_{3}$. Our 1D results are reported for two inversion coordinates. The full vibrational configuration interaction (FVCI) values for the complete set of normal modes were obtained using fourth-order and sixth-order expansions (Refs. 21, 60, and 11). All quantities are in a.u.

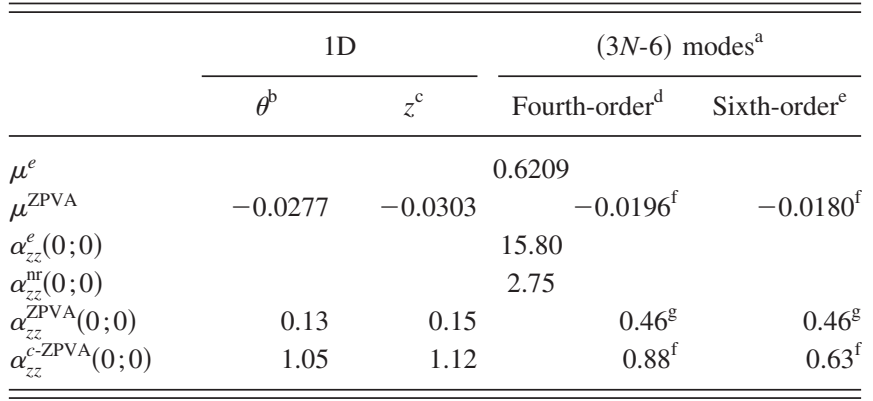

${ }^{\mathrm{a}}$ Data taken from Ref. 21.

${ }^{\mathrm{b}} \theta$ inversion coordinate $1 \mathrm{D}$ PES.

${ }^{c} z$ inversion coordinate $1 \mathrm{D}$ PES

${ }^{\mathrm{d}}$ V4M4T (Refs. 21, 60, and 11).

${ }^{\mathrm{e}} \mathrm{V} 4 \mathrm{M} 4 \mathrm{~T}+\mathrm{V} 2 \mathrm{M} 6 \mathrm{~T}$ (Refs. 21, 60, and 11)

${ }^{\mathrm{f}} \mu 3 \mathrm{M} 3 \mathrm{~T}+\mu 1 \mathrm{M} 4 \mathrm{~T}$ (Refs. 21, 60, and 11).

${ }^{\mathrm{g}} \alpha 2 \mathrm{M} 2 \mathrm{~T}+\alpha 1 \mathrm{M} 4 \mathrm{~T}$ (Refs. 21,60 , and 11).

erties, low-order expansions of the PES did not lead to converged vibrational NLO properties. In particular, there was a big jump in the vibrational static first and second hyperpolarizability upon going from a quartic to a sextic expansion for the PES. Thus, we previously concluded that the PES should not be expanded as a power series in terms of the normal coordinate associated with a single potential well. ${ }^{21}$ The procedure used in this paper solves this convergence problem. A comparison between the results shown in the second and third columns of Tables III-V with the data presented in the last two columns confirms that the sixth-order expansion is seriously flawed. It also suggests that the quartic expansion is satisfactory in this case.

The data presented in the second and third columns of Tables III-V show how the choice of inversion coordinate affects the static $P^{\mathrm{ZPVA}}$ and $P^{c \text {-ZPVA }}$ vibrational terms. It

TABLE IV. Electronic and vibrational contributions to the diagonal component of $\beta$ in the direction of $\mu$ for $\mathrm{NH}_{3}$. Our 1D results are reported for two inversion coordinates. The FVCI values for the complete set of $3 N-6$ normal modes were obtained using fourth-order and sixth-order expansions (Refs. 21, 60, and 11). All quantities are in a.u.

\begin{tabular}{|c|c|c|c|c|}
\hline & \multicolumn{2}{|c|}{$1 \mathrm{D}$} & \multicolumn{2}{|c|}{$(3 N-6) \operatorname{modes}^{\mathrm{a}}$} \\
\hline & $\theta^{b}$ & $z^{\mathrm{c}}$ & Fourth-order $^{\mathrm{d}}$ & Sixth-order ${ }^{\mathrm{e}}$ \\
\hline$\beta_{z z z}^{e}(0 ; 0,0)$ & & & -31.1 & \\
\hline$\beta_{z z z}^{\mathrm{nr}}(0 ; 0,0)$ & & & -115.1 & \\
\hline$\beta_{z z z}^{\mathrm{ZPVA}}(0 ; 0,0)$ & 0.8 & 0.8 & $-0.0^{\mathrm{f}}$ & $-0.0^{\mathrm{f}}$ \\
\hline$\beta_{z z z}^{c-\mathrm{ZPVA}}(0 ; 0,0)$ & -111.3 & -119.4 & $-114.6^{\mathrm{g}}$ & $-60.9^{\mathrm{g}}$ \\
\hline$\beta_{z z z}^{\text {nr }}(-\omega ; \omega, 0)_{\omega \rightarrow \infty}$ & & & -11.1 & \\
\hline$\beta_{z z z}^{c-\mathrm{ZPVA}}(-\omega ; \omega, 0)_{\omega \rightarrow \infty}$ & -3.7 & -3.6 & $-1.4^{\mathrm{h}}$ & $-0.5^{\mathrm{h}}$ \\
\hline
\end{tabular}

${ }^{\mathrm{a}}$ Data taken from Ref. 21.

${ }^{\mathrm{b}} \theta$ inversion coordinate 1D PES.

${ }^{c} z$ inversion coordinate $1 \mathrm{D}$ PES

${ }^{\mathrm{d}} \mathrm{V} 4 \mathrm{M} 4 \mathrm{~T}$ (Refs. 21, 60, and 11).

${ }^{\mathrm{e}} \mathrm{V} 4 \mathrm{M} 4 \mathrm{~T}+\mathrm{V} 2 \mathrm{M} 6 \mathrm{~T}$ (Refs. 21, 60, and 11)

${ }^{\mathrm{f}} \beta 2 \mathrm{M} 2 \mathrm{~T}+\beta 1 \mathrm{M} 4 \mathrm{~T}$ (Refs. 21,60 , and 11).

${ }^{\mathrm{g}} \mu 3 \mathrm{M} 3 \mathrm{~T}+\mu 1 \mathrm{M} 4 \mathrm{~T}$ (Refs. 21,60 , and 11).

${ }^{\mathrm{h}} \alpha 2 \mathrm{M} 2 \mathrm{~T}+\alpha 1 \mathrm{M} 4 \mathrm{~T}$ (Refs. 21, 60, and 11).
TABLE V. Electronic and vibrational contributions to the diagonal component of $\gamma$ in the direction of $\mu$ for $\mathrm{NH}_{3}$. Our 1D results are reported for two inversion coordinates. The FVCI values for the complete set of $3 N-6$ normal modes were obtained using fourth-order and sixth-order expansions (Refs. 21, 60, and 11). All quantities are in a.u.

\begin{tabular}{|c|c|c|c|c|}
\hline & \multicolumn{2}{|c|}{$1 \mathrm{D}$} & \multicolumn{2}{|c|}{$(3 N-6) \operatorname{modes}^{\mathrm{a}}$} \\
\hline & $\theta^{\mathrm{b}}$ & $z^{\mathrm{c}}$ & Fourth-order $^{\mathrm{d}}$ & Sixth-order ${ }^{\mathrm{e}}$ \\
\hline$\gamma_{z z z z}^{\mathcal{e}}(0 ; 0,0,0)$ & & & 7082 & \\
\hline$\gamma_{z z z z}^{\mathrm{nr}}(0 ; 0,0,0)$ & & & 7792 & \\
\hline$\gamma_{z z z z}^{\mathrm{ZPVA}}(0 ; 0,0,0)$ & 281 & 306 & $173^{f}$ & $158^{f}$ \\
\hline$\gamma_{z z z z}^{c \text {-ZPVA }}(0 ; 0,0,0)$ & 25234 & 26430 & $25041^{\mathrm{g}}$ & $9504^{\mathrm{g}}$ \\
\hline$\gamma_{z z z z}^{\mathrm{nr}}(-\omega ; \omega, 0,0)_{\omega \rightarrow \infty}$ & & & 527 & \\
\hline$\gamma_{z z z z}^{c \text {-ZPVA }}(-\omega ; \omega, 0,0)_{\omega \rightarrow \infty}$ & 264 & 260 & $288^{\mathrm{h}}$ & $137^{\mathrm{h}}$ \\
\hline$\gamma_{z z z z}^{\mathrm{nr}}(-2 \omega ; \omega, \omega, 0)_{\omega \rightarrow \infty}$ & & & 32 & \\
\hline$\gamma_{z z z z}^{c-Z P V A}(-2 \omega ; \omega, \omega, 0)_{\omega \rightarrow \infty}$ & -46 & -48 & $11^{\mathrm{i}}$ & $19^{\mathrm{i}}$ \\
\hline \multicolumn{5}{|c|}{ 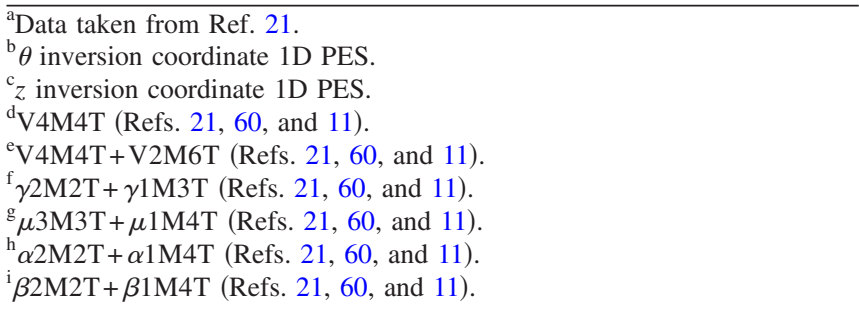 } \\
\hline
\end{tabular}

turns out that the sensitivity is low with maximum differences of about $7 \%$ in $P^{c \text {-ZPVA }}$ (the absolute magnitudes of the ZPVA differences are too small to be noteworthy). This good agreement suggests that the precise definition of the coordinates used to describe the anharmonic vibrations in reduced dimensionality calculations may not be a critical issue. Of course, further studies of this point are necessary in order to derive a definitive conclusion.

We did investigate the origin of the difference between the $\theta$ and $z$ NLO properties by combining the 1D effective potential for the $\theta$ coordinate with all subsequent vibrational NLO calculations done using the $z$ coordinate. The results for these "mixed" calculations turned out to be very close to the "pure" values obtained using the $z$ coordinate for the entire treatment. For instance the mixed first and second static $c$-ZPVA hyperpolarizabilities were -120.6 and 26441 a.u., respectively, compared to the corresponding pure values of -119.6 and 26430 a.u. Thus, the vibrational NLO differences between the $\theta$ and $z$ coordinates is determined primarily by the kinetic energy rather than the potential energy.

Our new treatment fully incorporates the effect of tunneling which, however, is insignificant for ammonia. This can be seen from the fact that (i) a field as small as 0.0004 a.u. is well into the strong field regime and (ii) the difference between the (+) and (-) properties in Eq. (11) is entirely negligible. Of course, the situation will depend in general upon the barrier height and the temperature, which has not been considered here.

The lowering of the energy barrier will also radically increase the problems of the one minima approach to describe the inversion anharmonicity. Indeed as we have discussed above, the large differences between quartic and sextic one minimum Taylor expansion indicate that already for $\mathrm{NH}_{3}$ the one-well model may not be reliable. 


\section{SUMARY AND FUTURE OUTLOOK}

We have taken the first steps toward a general reduced dimensionality treatment of vibrational NLO for molecules where the primary contributions arise from a limited number of nuclear motions, some or all of which may exhibit large anharmonic effects. Our treatment is based on a FF-NR approach wherein the key motions are decoupled from all other vibrations in first order. In this paper the focus is on large amplitude anharmonic vibrations. The umbrella motion in ammonia is used as an example and the quasidegeneracy of the ground vibrational state is explicitly taken into account. Vibrational wave functions for the inversion motion, which are required for the FF-NR treatment, are obtained by numerical solution of the 1D Schrodinger equations including a proper expression for the kinetic energy operator. We find that our method overcomes convergence problems associated with a conventional single well treatment. The calculations confirm that it is essential to consider the vibrational contribution to NLO properties in $\mathrm{NH}_{3}$ and that the umbrella mode is dominant in this regard. It turns out that the particular definition of the inversion coordinate is not very important. In addition, for $\mathrm{NH}_{3}$ the effect of tunneling is insignificant.

There is much more to be done in order to further develop and validate the methodology presented here. We want to examine other anharmonic potentials and cases where there is more than one key anharmonic mode. The dimers of hydrogen fluoride and water might be especially interesting in this connection since it is known that BK-PT is nonconvergent for these species. ${ }^{61,62}$ One of our major goals is to apply this treatment to endohedral and other fullerenes, ${ }^{3}$ which we expect will have large NLO properties arising from large amplitude anharmonic vibrations.

\section{ACKNOWLEDGMENTS}

Financial help was furnished by the European Union under Grant No. MTKD-CT2006-042488 and the Spanish government under Grant No. CTQ2008-06696/BQU programs.

${ }^{1}$ K. Bouchouit, Z. Essaidi, S. Abed, A. Migalska-Zalas, B. Derkowska, N. Benali-Cherif, M. Mihaly, A. Meghea, and B. Sahraoui, Chem. Phys. Lett. 455, 270 (2008).

${ }^{2}$ P. N. Butcher and D. Cotter, in The Elements of Nonlinear Optics: Cambridge Studies in Modern Optics, edited by P. L. Night and W. J. Firth (Cambridge University Press, Cambridge, 1990).

${ }^{3}$ O. Loboda, R. Zalesny, A. Avramopoulos, J. M. Luis, B. Kirtman, N. Tagmatarchis, H. Reis, and M. G. Papadopoulos, J. Phys. Chem. A 113 1159 (2009)

${ }^{4}$ E. Santiago, M. A. Castro, T. L. Fonseca, and P. K. Mukherjee, J. Chem. Phys. 128, 064310 (2008).

${ }^{5}$ A. J. Thorvaldsen, K. Ruud, and M. Jaszunski, J. Phys. Chem. A 112, 11942 (2008).

${ }^{6}$ C. C. Chou and B. Y. Jin, Theor. Chem. Acc. 122, 313 (2009).

${ }^{7}$ D. M. Bishop and B. Kirtman, J. Chem. Phys. 95, 2646 (1991)

${ }^{8}$ D. M. Bishop and B. Kirtman, J. Chem. Phys. 97, 5255 (1992).

${ }^{9}$ D. M. Bishop, J. M. Luis, and B. Kirtman, J. Chem. Phys. 108, 10013 (1998)

${ }^{10}$ O. Christiansen, J. Chem. Phys. 122, 194105 (2005).

${ }^{11}$ O. Christiansen, J. Kongsted, M. J. Paterson, and J. M. Luis, J. Chem. Phys. 125, 214309 (2006).

${ }^{12}$ D. M. Bishop, M. Hasan, and B. Kirtman, J. Chem. Phys. 103, 4157 (1995).

${ }^{13}$ J. M. Luis, M. Duran, and J. L. Andres, J. Chem. Phys. 107, 1501
(1997).

${ }^{14}$ J. M. Luis, J. Marti, M. Duran, J. L. Andres, and B. Kirtman, J. Chem. Phys. 108, 4123 (1998).

${ }^{15}$ B. Kirtman, J. M. Luis, and D. M. Bishop, J. Chem. Phys. 108, 10008 (1998).

${ }^{16}$ B. Kirtman and J. M. Luis, J. Chem. Phys. 128, 114101 (2008).

${ }^{17}$ J. M. Luis, M. Duran, J. L. Andres, B. Champagne, and B. Kirtman, J. Chem. Phys. 111, 875 (1999).

${ }^{18}$ M. Torrent-Sucarrat, M. Sola, M. Duran, J. M. Luis, and B. Kirtman, J. Chem. Phys. 118, 711 (2003).

${ }^{19}$ M. Torrent-Sucarrat, M. Sola, M. Duran, J. M. Luis, and B. Kirtman, J. Chem. Phys. 116, 5363 (2002).

${ }^{20}$ B. Champagne, J. M. Luis, M. Duran, J. L. Andres, and B. Kirtman, J. Chem. Phys. 112, 1011 (2000).

${ }^{21}$ J. M. Luis, M. Torrent-Sucarrat, O. Christiansen, and B. Kirtman, J. Chem. Phys. 127, 084118 (2007).

${ }^{22}$ M. Torrent-Sucarrat, M. Sola, M. Duran, J. M. Luis, and B. Kirtman, J. Chem. Phys. 120, 6346 (2004).

${ }^{23}$ J. M. Luis, M. Duran, and B. Kirtman, J. Chem. Phys. 115, 4473 (2001).

${ }^{24}$ J. M. Luis, B. Champagne, and B. Kirtman, Int. J. Quantum Chem. 80, 471 (2000).

${ }^{25}$ M. Torrent-Sucarrat, J. M. Luis, and B. Kirtman, J. Chem. Phys. 122, 204108 (2005).

${ }^{26}$ J. H. Van Vleck, Phys. Rev. 33, 467 (1929).

${ }^{27}$ B. Kirtman, J. Chem. Phys. 49, 3890 (1968).

${ }^{28}$ B. Kirtman, J. Chem. Phys. 75, 798 (1981).

${ }^{29}$ K. Yagi, S. Hirata, and K. Hirao, Phys. Chem. Chem. Phys. 10, 1781 (2008).

${ }^{30}$ D. M. Bishop and E. K. Dalskov, J. Chem. Phys. 104, 1004 (1996).

${ }^{31}$ O. Quinet and B. Champagne, J. Chem. Phys. 109, 10594 (1998).

${ }^{32}$ N. C. Handy, S. Carter, and S. M. Colwell, Mol. Phys. 96, 477 (1999).

${ }^{33}$ N. Aquino, G. Campoy, and H. Yee-Madeira, Chem. Phys. Lett. 296, 111 (1998).

${ }^{34}$ A. Niño and C. Muñoz-Caro, Computers Chem. 18, 27 (1994).

${ }^{35}$ A. Niño and C. Muñoz-Caro, Computers Chem. 19, 371 (1995).

${ }^{36}$ D. Lauvergnat and A. Nauts, J. Chem. Phys. 116, 8560 (2002).

${ }^{37}$ D. Lauvergnat, E. Baloitcha, G. Dive, and M. Desouter-Lecomte, Chem. Phys. 326, 500 (2006).

${ }^{38}$ X. Chapuisat, A. Nauts, and J. P. Brunet, Mol. Phys. 72, 1 (1991).

${ }^{39}$ E. B. Wilson, Jr., J. C. Decius, and P. C. Cross, Molecular Vibrations (McGraw-Hill, New York, 1955).

${ }^{40}$ M. L. Senent, Chem. Phys. Lett. 296, 299 (1998).

${ }^{41}$ D. J. Rush and K. B. Wiberg, J. Phys. Chem. A 101, 3143 (1997).

${ }^{42}$ F. Gatti, Y. Justum, M. Menou, A. Nauts, and X. Chapuisat, J. Mol. Spectrosc. 181, 403 (1997).

${ }^{43}$ M. A. Harthcock and J. Laane, J. Mol. Spectrosc. 91, 300 (1982).

${ }^{44}$ W. H. Press, S. A. Teukolsky, W. T. Vetterling, and B. P. Flannery, $N u-$ merical Recipes (Cambridge University Press, Cambridge, 1992).

${ }^{45}$ M. J. Frisch, G. W. Trucks, H. B. Schlegel et al., Gaussian 03, Revision C.02, Gaussian, Inc., Wallingford CT, 2004.

${ }^{46}$ A. J. Sadlej, Collect. Czech. Chem. Commun. 53, 1995 (1988).

${ }^{47}$ A. Avramopoulos, H. Reis, J. B. Li, and M. G. Papadopoulos, J. Am. Chem. Soc. 126, 6179 (2004).

${ }^{48}$ V. E. Ingamells, M. G. Papadopoulos, and S. G. Raptis, Chem. Phys. Lett. 307, 484 (1999).

${ }^{49}$ M. Urban and A. J. Sadlej, J. Chem. Phys. 103, 9692 (1995).

${ }^{50}$ T. Pluta and A. J. Sadlej, Chem. Phys. Lett. 297, 391 (1998).

${ }^{51}$ T. Pluta and A. J. Sadlej, J. Chem. Phys. 114, 136 (2001).

${ }^{52}$ V. Spirko, J. Mol. Spectrosc. 101, 30 (1983).

${ }^{53}$ T. H. Dunning, J. Chem. Phys. 90, 1007 (1989).

${ }^{54}$ R. A. Kendall, T. H. Dunning, and R. J. Harrison, J. Chem. Phys. 96, 6796 (1992)

${ }^{55}$ J. Pesonen, A. Miani, and L. Halonen, J. Chem. Phys. 115, 1243 (2001).

${ }^{56}$ T. Rajamaki, A. Miani, and L. Halonen, J. Chem. Phys. 118, 10929 (2003).

${ }^{57}$ T. Rajamaki, A. Miani, and L. Halonen, J. Chem. Phys. 118, 6358 (2003).

${ }^{58}$ S. N. Yurchenko, J. G. Zheng, H. Lin, P. Jensen, and W. Thiel, J. Chem. Phys. 123, 134308 (2005).

${ }^{59}$ P. J. Davis and P. Rabinowitz, Numerical Integration (Blaisdell, London, 
1967), p. 166.

${ }^{60}$ These truncations are denoted as $X m M t T$, where $X$ may be the potential energy (V) or the electronic contribution to the static electrical property, $m$ is the maximum number of coupled modes, and $t$ is the highest power in the expansion ( $M$ and $T$ are included for cosmetic reasons only).

${ }^{61}$ D. M. Bishop, J. Pipin, and B. Kirtman, J. Chem. Phys. 102, 6778 (1995).

${ }^{62}$ U. Eckart and A. J. Sadlej, Mol. Phys. 99, 735 (2001). 